\title{
HERMITE-HADAMARD TYPE INEQUALITIES FOR THE PRODUCT OF $(\alpha, m)$-CONVEX FUNCTIONS
}

\author{
HONG-PING YIN AND FENG QI
}

ABstract. In the paper, the authors establish some Hermite-Hadamard type inequalities for the product of two $(\alpha, m)$-convex functions.

\section{INTRODUCTION}

The following definitions are well-known in the literature.

Definition 1.1. A function $f: I \subseteq \mathbb{R}=(-\infty, \infty) \rightarrow \mathbb{R}$ is said to be convex if

$$
f(\lambda x+(1-\lambda) y) \leq \lambda f(x)+(1-\lambda) f(y)
$$

holds for all $x, y \in I$ and $\lambda \in[0,1]$.

Definition $1.2([7])$. For $f:[0, b] \rightarrow \mathbb{R}$ and $m \in(0,1]$, if

$$
f(t x+m(1-t) y) \leq t f(x)+m(1-t) f(y)
$$

is valid for all $x, y \in[0, b]$ and $t \in[0,1]$, then we say that $f(x)$ is $m$-convex on $[0, b]$.

Definition $1.3([4])$. For $f:[0, b] \rightarrow \mathbb{R}$ and $(\alpha, m) \in(0,1] \times(0,1]$, if

$$
f(t x+m(1-t) y) \leq t^{\alpha} f(x)+m\left(1-t^{\alpha}\right) f(y)
$$

is valid for all $x, y \in[0, b]$ and $t \in[0,1]$, then we say that $f(x)$ is $(\alpha, m)$ convex on $[0, b]$.

In recent decades, many inequalities of Hermite-Hadamard type for various kinds of convex functions have been established. Some of them may be recited as follows.

Theorem 1.4 ([3]). Let $f:[a, b] \subseteq \mathbb{R}_{0}=[0, \infty) \rightarrow \mathbb{R}$ be $m$-convex for fixed $m \in(0,1]$. Then

$$
\frac{1}{b-a} \int_{a}^{b} f(x) d x \leq \min \left\{\frac{f(a)+m f(b / m)}{2}, \frac{m f(a / m)+f(b)}{2}\right\} .
$$




\section{H.-P. YIN AND F. QI}

Theorem 1.5 ([5]). Let $f, g:[a, b] \subseteq \mathbb{R} \rightarrow \mathbb{R}_{0}$ be convex functions. Then

$$
\frac{1}{b-a} \int_{a}^{b} f(x) g(x) d x \leq \frac{1}{3} M(a, b)+\frac{1}{6} N(a, b)
$$

where $M(a, b)=f(a) g(a)+f(b) g(b)$ and $N(a, b)=f(a) g(b)+f(b) g(a)$.

Theorem $1.6([2])$. Let $f, g: \mathbb{R}_{0} \rightarrow \mathbb{R}_{0}$ satisfy $f g \in L([a, b])$, where $0 \leq$ $a<b<\infty$. If $f$ is $m_{1}$-convex and $g$ is $m_{2}$-convex on $[a, b]$ for some fixed $m_{1}, m_{2} \in(0,1]$, then

$$
\frac{1}{b-a} \int_{a}^{b} f(x) g(x) d x \leq \min \left\{M_{1}, M_{2}\right\}
$$

where

$$
\begin{aligned}
M_{1}= & \frac{1}{3}\left[f(a) g(a)+m_{1} m_{2} f\left(\frac{b}{m_{1}}\right) g\left(\frac{b}{m_{2}}\right)\right] \\
& +\frac{1}{6}\left[m_{2} f(a) g\left(\frac{b}{m_{2}}\right)+m_{1} f\left(\frac{b}{m_{1}}\right) g(a)\right]
\end{aligned}
$$

and

$$
\begin{aligned}
M_{2}= & \frac{1}{3}\left[f(b) g(b)+m_{1} m_{2} f\left(\frac{a}{m_{1}}\right) g\left(\frac{a}{m_{2}}\right)\right] \\
& +\frac{1}{6}\left[m_{1} f\left(\frac{a}{m_{1}}\right) g(b)+m_{2} f(b) g\left(\frac{a}{m_{2}}\right)\right] .
\end{aligned}
$$

Theorem 1.7 ([2]). Let $f, g: \mathbb{R}_{0} \rightarrow \mathbb{R}_{0}$ satisfy $f g \in L([a, b])$ with $0 \leq$ $a<b<\infty$. If $f$ is $\left(\alpha_{1}, m_{1}\right)$-convex and $g$ is $\left(\alpha_{2}, m_{2}\right)$-convex on $[a, b]$ for $\left(\alpha_{1}, m_{1}\right),\left(\alpha_{2}, m_{2}\right) \in(0,1] \times(0,1]$, then

$$
\frac{1}{b-a} \int_{a}^{b} f(x) g(x) d x \leq \min \left\{N_{1}, N_{2}\right\}
$$

where

$$
\begin{aligned}
N_{1}= & \frac{f(a) g(a)}{\alpha_{1}+\alpha_{2}+1}+m_{2}\left[\frac{1}{\alpha_{1}+1}-\frac{1}{\alpha_{1}+\alpha_{2}+1}\right] f(a) g\left(\frac{b}{m_{2}}\right) \\
& -m_{1}\left(\frac{1}{\alpha_{1}+\alpha_{2}+1}-\frac{1}{\alpha_{2}+1}\right) g(a) f\left(\frac{b}{m_{1}}\right) \\
& +m_{1} m_{2}\left(1-\frac{1}{\alpha_{1}+1}-\frac{1}{\alpha_{2}+1}+\frac{1}{\alpha_{1}+\alpha_{2}+1}\right) f\left(\frac{b}{m_{1}}\right) g\left(\frac{b}{m_{2}}\right)
\end{aligned}
$$




\section{HERMITE-HADAMARD INEQUALITIES FOR CONVEX FUNCTIONS}

and

$$
\begin{aligned}
N_{2}= & \frac{f(b) g(b)}{\alpha_{1}+\alpha_{2}+1}+m_{2}\left[\frac{1}{\alpha_{1}+1}-\frac{1}{\alpha_{1}+\alpha_{2}+1}\right] f(b) g\left(\frac{a}{m_{2}}\right) \\
& -m_{1}\left(\frac{1}{\alpha_{1}+\alpha_{2}+1}-\frac{1}{\alpha_{2}+1}\right) g(b) f\left(\frac{a}{m_{1}}\right) \\
& +m_{1} m_{2}\left(1-\frac{1}{\alpha_{1}+1}-\frac{1}{\alpha_{2}+1}+\frac{1}{\alpha_{1}+\alpha_{2}+1}\right) f\left(\frac{a}{m_{1}}\right) g\left(\frac{a}{m_{2}}\right) .
\end{aligned}
$$

In recent years, some inequalities of Hermite-Hadamard type for other kinds of convex functions were created in, $[1,6,8,9,10,11,12]$ and closely related references therein.

The aim of this paper is to present some new inequalities of HermiteHadamard type for the product of two $(\alpha, m)$-convex functions, which generalizes those results mentioned above.

\section{Main Results}

We are now in a position to establish some new integral inequalities of Hermite-Hadamard type for the product of two $(\alpha, m)$-convex functions.

Theorem 2.1. Let $f, g: \mathbb{R}_{0} \rightarrow \mathbb{R}_{0}$ satisfy $f, f g^{q} \in L([a, b])$, where $0 \leq a<$ $b<\infty$ and $q \geq 1$. If $f$ is $\left(\alpha_{1}, m_{1}\right)$-convex on $\left[0, \frac{b}{m_{1}}\right]$ and $g^{q}$ is $\left(\alpha_{2}, m_{2}\right)$ convex on $\left[0, \frac{b}{m_{2}}\right]$ for $\left(\alpha_{1}, m_{1}\right),\left(\alpha_{2}, m_{2}\right) \in(0,1] \times(0,1]$, then

$$
\begin{aligned}
& \frac{1}{b-a} \int_{a}^{b} f(x) g(x) d x \\
& \leq \frac{\left[N\left(a, b ; f, \alpha_{1}, m_{1}\right)\right]^{1-1 / q} \min \left\{\left[M\left(a, b ; f, g^{q}\right)\right]^{1 / q},\left[M\left(b, a ; f, g^{q}\right)\right]^{1 / q}\right\}}{\left(\alpha_{1}+1\right)\left[\left(\alpha_{2}+1\right)\left(\alpha_{1}+\alpha_{2}+1\right)\right]^{1 / q}} .
\end{aligned}
$$

where

$$
N(a, b ; f, \alpha, m)=f(a)+\alpha m f\left(\frac{b}{m}\right)
$$

and

$$
\begin{gathered}
M(a, b ; f, g)=\left(\alpha_{1}+1\right)\left(\alpha_{2}+1\right) f(a) g(a)+\alpha_{2} m_{2}\left(\alpha_{2}+1\right) f(a) g\left(\frac{b}{m_{2}}\right) \\
+\alpha_{1} m_{1}\left(\alpha_{1}+1\right) g(a) f\left(\frac{b}{m_{1}}\right)+\alpha_{1} \alpha_{2}\left(\alpha_{1}+\alpha_{2}+2\right) m_{1} m_{2} f\left(\frac{b}{m_{1}}\right) g\left(\frac{b}{m_{2}}\right) .
\end{gathered}
$$

MISSOURI J. OF MATH. SCI., FALL 2015 


\section{H.-P. YIN AND F. QI}

Proof. Letting $x=t a+(1-t) b$ for $t \in[0,1]$ and making use of Hölder's integral inequality yields

$$
\begin{aligned}
& \frac{1}{b-a} \int_{a}^{b} f(x) g(x) d x=\int_{0}^{1} f(t a+(1-t) b) g(t a+(1-t) b) d t \\
\leq & {\left[\int_{0}^{1} f(t a+(1-t) b) d t\right]^{1-1 / q}\left[\int_{0}^{1} f(t a+(1-t) b) g^{q}(t a+(1-t) b) d t\right]^{1 / q} . }
\end{aligned}
$$

Further employing the conditions that $f$ is $\left(\alpha_{1}, m_{1}\right)$-convex on $\left[0, \frac{b}{m_{1}}\right]$ and $g^{q}$ is $\left(\alpha_{2}, m_{2}\right)$-convex on $\left[0, \frac{b}{m_{2}}\right]$ leads to

$$
\begin{aligned}
\int_{0}^{1} f(t a+(1-t) b) d t & \leq \int_{0}^{1}\left[t^{\alpha_{1}} f(a)+m_{1}\left(1-t^{\alpha_{1}}\right) f\left(\frac{b}{m_{1}}\right)\right] d t \\
& =\frac{1}{\alpha_{1}+1} N\left(a, b ; f, \alpha_{1}, m_{1}\right)
\end{aligned}
$$

and

$$
\begin{aligned}
& \int_{0}^{1} f(t a+(1-t) b) g^{q}(t a+(1-t) b) d t \\
& \leq \int_{0}^{1}\left[t^{\alpha_{1}} f(a)+m_{1}\left(1-t^{\alpha_{1}}\right) f\left(\frac{b}{m_{1}}\right)\right]\left[t^{\alpha_{2}} g^{q}(a)+m_{2}\left(1-t^{\alpha_{2}}\right) g^{q}\left(\frac{b}{m_{2}}\right)\right] d t \\
& =\frac{1}{\alpha_{1}+\alpha_{2}+1} f(a) g^{q}(a)+\frac{\alpha_{2} m_{2}}{\left(\alpha_{1}+1\right)\left(\alpha_{1}+\alpha_{2}+1\right)} f(a) g^{q}\left(\frac{b}{m_{2}}\right) \\
& +\frac{\alpha_{1} m_{1}}{\left(\alpha_{2}+1\right)\left(\alpha_{1}+\alpha_{2}+1\right)} f\left(\frac{b}{m_{1}}\right) g^{q}(a) \\
& +\frac{\alpha_{1} \alpha_{2}\left(\alpha_{1}+\alpha_{2}+2\right) m_{1} m_{2}}{\left(\alpha_{1}+1\right)\left(\alpha_{2}+1\right)\left(\alpha_{1}+\alpha_{2}+1\right)} f\left(\frac{b}{m_{1}}\right) g^{q}\left(\frac{b}{m_{2}}\right) \\
& =\frac{1}{\left(\alpha_{1}+1\right)\left(\alpha_{2}+1\right)\left(\alpha_{1}+\alpha_{2}+1\right)} M\left(a, b ; f, g^{q}\right) .
\end{aligned}
$$

The proof of Theorem 2.1 is complete.

Remark 2.2. Theorem 2.1 applied to $q=1$ becomes the inequality (1.7).

Corollary 2.3. Under conditions of Theorem 2.1,

(1) if $\alpha_{1}=\alpha_{2}=\alpha$, we have

$$
\begin{aligned}
& \frac{1}{b-a} \int_{a}^{b} f(x) g(x) d x \\
& \leq \frac{\left[N\left(a, b ; f, \alpha, m_{1}\right)\right]^{1-1 / q} \min \left\{\left[M\left(a, b ; f, g^{q}\right)\right]^{1 / q},\left[M\left(b, a ; f, g^{q}\right)\right]^{1 / q}\right\}}{(\alpha+1)^{1+1 / q}(2 \alpha+1)^{1 / q}}
\end{aligned}
$$




\section{HERMITE-HADAMARD INEQUALITIES FOR CONVEX FUNCTIONS}

(2) if $m_{1}=m_{2}=m$, we have

$$
\begin{aligned}
& \frac{1}{b-a} \int_{a}^{b} f(x) g(x) d x \\
& \leq \frac{\left[N\left(a, b ; f, \alpha_{1}, m\right)\right]^{1-1 / q} \min \left\{\left[M\left(a, b ; f, g^{q}\right)\right]^{1 / q},\left[M\left(b, a ; f, g^{q}\right)\right]^{1 / q}\right\}}{\left(\alpha_{1}+1\right)\left[\left(\alpha_{2}+1\right)\left(\alpha_{1}+\alpha_{2}+1\right)\right]^{1 / q}}
\end{aligned}
$$

(3) if $\alpha_{1}=\alpha_{2}=m_{1}=m_{2}=1$, we have

$$
\begin{aligned}
\frac{1}{b-a} \int_{a}^{b} f(x) & g(x) d x \leq \frac{1}{2}\left(\frac{1}{3}\right)^{1 / q}[f(a)+f(b)]^{1-1 / q} \\
\times & {\left[2 f(a) g^{q}(a)+f(a) g^{q}(b)+f(b) g^{q}(a)+2 f(b) g^{q}(b)\right]^{1 / q} . }
\end{aligned}
$$

Theorem 2.4. Let $f, g: \mathbb{R}_{0} \rightarrow \mathbb{R}_{0}$ be such that $f^{q}, g^{q /(q-1)} \in L([a, b])$, where $0 \leq a<b<\infty$ and $q>1$. If $f^{q}$ is $\left(\alpha_{1}, m_{1}\right)$-convex on $\left[0, \frac{b}{m_{1}}\right]$ and $g^{q /(q-1)}$ is $\left(\alpha_{2}, m_{2}\right)$-convex on $\left[0, \frac{b}{m_{2}}\right]$ for $\left(\alpha_{1}, m_{1}\right),\left(\alpha_{2}, m_{2}\right) \in(0,1] \times(0,1]$, then

$$
\begin{aligned}
& \frac{1}{b-a} \int_{a}^{b} f(x) g(x) d x \leq\left[\frac{\min \left\{N\left(a, b ; f^{q}, \alpha_{1}, m_{1}\right), N\left(b, a ; f^{q}, \alpha_{1}, m_{1}\right)\right\}}{\alpha_{1}+1}\right]^{1 / q} \\
& \times\left[\frac{\min \left\{N\left(a, b ; g^{q /(q-1)}, \alpha_{2}, m_{2}\right), N\left(b, a ; g^{q /(q-1)}, \alpha_{2}, m_{2}\right)\right\}}{\alpha_{2}+1}\right]^{1-1 / q},
\end{aligned}
$$

where $N(a, b ; f, \alpha, m)$ is defined by (2.1).

Proof. Taking $x=t a+(1-t) b$ for $t \in[0,1]$ and using Hölder's integral inequality generates

$$
\begin{aligned}
& \frac{1}{b-a} \int_{a}^{b} f(x) g(x) d x=\int_{0}^{1} f(t a+(1-t) b) g(t a+(1-t) b) d t \\
& \quad \leq\left[\int_{0}^{1} f^{q}(t a+(1-t) b) d t\right]^{1 / q}\left[\int_{0}^{1} g^{q /(q-1)}(t a+(1-t) b) d t\right]^{1-1 / q} .
\end{aligned}
$$

Utilizing properties that $f^{q}$ is $\left(\alpha_{1}, m_{1}\right)$-convex on $\left[0, \frac{b}{m_{1}}\right]$ and that $g^{q /(q-1)}$ is $\left(\alpha_{2}, m_{2}\right)$-convex on $\left[0, \frac{b}{m_{2}}\right]$ discovers

$$
\begin{aligned}
\int_{0}^{1} f^{q}(t a+(1-t) b) d t & \leq \int_{0}^{1}\left[t^{\alpha_{1}} f^{q}(a)+m_{1}\left(1-t^{\alpha_{1}}\right) f^{q}\left(\frac{b}{m_{1}}\right)\right] d t \\
& =\frac{1}{\alpha_{1}+1} N\left(a, b ; f^{q}, \alpha_{1}, m_{1}\right) .
\end{aligned}
$$

MISSOURI J. OF MATH. SCI., FALL 2015 


\section{H.-P. YIN AND F. QI}

Considering the symmetry of the estimated definite integral with respect to $a$ and $b$ results in

$$
\int_{0}^{1} f^{q}(t a+(1-t) b) d t \leq \frac{\min \left\{N\left(a, b ; f^{q}, \alpha_{1}, m_{1}\right), N\left(b, a ; f^{q}, \alpha_{1}, m_{1}\right)\right\}}{\alpha_{1}+1} .
$$

Similarly, we have

$$
\begin{aligned}
& \int_{0}^{1} g^{q /(q-1)}(t a+(1-t) b) d t \\
& \leq \frac{\min \left\{N\left(a, b ; g^{q /(q-1)}, \alpha_{2}, m_{2}\right), N\left(b, a ; g^{q /(q-1)}, \alpha_{2}, m_{2}\right\}\right.}{\alpha_{2}+1} .
\end{aligned}
$$

Theorem 2.4 is thus proved.

Corollary 2.5. Under conditions of Theorem 2.4, if $\alpha_{1}=\alpha_{2}=m_{1}=$ $m_{2}=1$, then

$$
\frac{1}{b-a} \int_{a}^{b} f(x) g(x) d x \leq \frac{\left[f^{q}(a)+f^{q}(b)\right]^{1 / q}\left[g^{q /(q-1)}(a)+g^{q /(q-1)}(b)\right]^{1-1 / q}}{2} .
$$

Theorem 2.6. Let $f, g: \mathbb{R}_{0} \rightarrow \mathbb{R}_{0}$ be such that $f^{p} g^{q-\ell(q-1)}, f^{(q-p) /(q-1)} g^{\ell} \in$ $L([a, b])$, where $0 \leq a<b<\infty, q>1, q>p>0$, and $\frac{q}{q-1}>\ell>0$. If $f^{p}$ and $f^{(q-p) /(q-1)}$ are $\left(\alpha_{1}, m_{1}\right)$-convex on $\left[0, \frac{b}{m_{1}}\right]$ and if $g^{\ell}$ and $g^{q-\ell /(q-1)}$ are $\left(\alpha_{2}, m_{2}\right)$-convex on $\left[0, \frac{b}{m_{2}}\right]$ for $\left(\alpha_{1}, m_{1}\right),\left(\alpha_{2}, m_{2}\right) \in(0,1] \times(0,1]$, then

$$
\begin{aligned}
& \frac{1}{b-a} \int_{a}^{b} f(x) g(x) d x \leq \frac{1}{\left(\alpha_{1}+1\right)\left(\alpha_{2}+1\right)\left(\alpha_{1}+\alpha_{2}+1\right)} \\
& \times\left[\min \left\{M\left(a, b ; f^{p}, g^{q-\ell(q-1)}\right), M\left(b, a ; f^{p}, g^{q-\ell(q-1)}\right)\right\}\right]^{1 / q} \\
& \times\left[\min \left\{M\left(a, b ; f^{(q-p) /(q-1)}, g^{\ell}\right), M\left(b, a ; f^{(q-p) /(q-1)}, g^{\ell}\right)\right\}\right]^{1-1 / q},
\end{aligned}
$$

where $M(a, b ; f, g)$ is defined by $(2.2)$.

Proof. Letting $x=t a+(1-t) b$ for $t \in[0,1]$ and using Hölder's integral inequality, we obtain

$$
\begin{aligned}
\frac{1}{b-a} \int_{a}^{b} f(x) g(x) d x=\int_{0}^{1} f(t a+(1-t) b) g(t a+(1-t) b) d t \\
\leq\left[\int_{0}^{1} f^{p}(t a+(1-t) b) g^{q-\ell(q-1)}(t a+(1-t) b) d t\right]^{1 / q} \\
\times\left[\int_{0}^{1} f^{(q-p) /(q-1)}(t a+(1-t) b) g^{\ell}(t a+(1-t) b) d t\right]^{1-1 / q} .
\end{aligned}
$$




\section{HERMITE-HADAMARD INEQUALITIES FOR CONVEX FUNCTIONS}

Further by virtue of properties that the function $f^{p}$ is $\left(\alpha_{1}, m_{1}\right)$-convex on $\left[0, \frac{b}{m_{1}}\right]$ and that the function $g^{q-\ell /(q-1)}$ is $\left(\alpha_{2}, m_{2}\right)$-convex on $\left[0, \frac{b}{m_{2}}\right]$, we have

$$
\begin{aligned}
\int_{0}^{1} f^{p}(t a+(1-t) b) g^{q-\ell(q-1)}(t a+(1-t) b) d t \\
\leq \int_{0}^{1}\left[t^{\alpha_{1}} f^{p}(a)+m_{1}\left(1-t^{\alpha_{1}}\right) f^{p}\left(\frac{b}{m_{1}}\right)\right] \\
\quad \times\left[t^{\alpha_{2}} g^{q-\ell(q-1)}(a)+m_{2}\left(1-t^{\alpha_{2}}\right) g^{q-\ell(q-1)}\left(\frac{b}{m_{2}}\right)\right] d t \\
=\frac{1}{\alpha_{1}+\alpha_{2}+1} f^{p}(a) g^{q-\ell(q-1)}(a) \\
\quad+\frac{\alpha_{2} m_{2}}{\left(\alpha_{1}+1\right)\left(\alpha_{1}+\alpha_{2}+1\right)} f^{p}(a) g^{q-\ell(q-1)}\left(\frac{b}{m_{2}}\right) \\
\quad+\frac{\alpha_{1} m_{1}}{\left(\alpha_{2}+1\right)\left(\alpha_{1}+\alpha_{2}+1\right)} f^{p}\left(\frac{b}{m_{1}}\right) g^{q-\ell(q-1)}(a) \\
\quad+\frac{\alpha_{1} \alpha_{2}\left(\alpha_{1}+\alpha_{2}+2\right) m_{1} m_{2}}{\left(\alpha_{1}+1\right)\left(\alpha_{2}+1\right)\left(\alpha_{1}+\alpha_{2}+1\right)} f^{p}\left(\frac{b}{m_{1}}\right) g^{q-\ell(q-1)}\left(\frac{b}{m_{2}}\right) \\
=\frac{1}{\left(\alpha_{1}+1\right)\left(\alpha_{2}+1\right)\left(\alpha_{1}+\alpha_{2}+1\right)} M\left(a, b ; f^{p}, g^{q-\ell(q-1)}\right) .
\end{aligned}
$$

Changing the order of $a$ and $b$ in the above arguments reveals

$$
\begin{aligned}
\int_{0}^{1} f^{p}(t a+(1-t) b) g^{q-\ell(q-1)}(t a+(1-t) b) d t \\
\leq \frac{\min \left\{M\left(a, b ; f^{p}, g^{q-\ell(q-1)}\right), M\left(b, a ; f^{p}, g^{q-\ell(q-1)}\right)\right\}}{\left(\alpha_{1}+1\right)\left(\alpha_{2}+1\right)\left(\alpha_{1}+\alpha_{2}+1\right)}
\end{aligned}
$$

and

$$
\begin{aligned}
\int_{0}^{1} f^{(q-p) /(q-1)}(t a+(1-t) b) g^{\ell}(t a+(1-t) b) d t \\
\leq \\
\leq \frac{\min \left\{M\left(a, b ; f^{(q-p) /(q-1)}, g^{\ell}\right), M\left(b, a ; f^{(q-p) /(q-1)}, g^{\ell}\right)\right\}}{\left(\alpha_{1}+1\right)\left(\alpha_{2}+1\right)\left(\alpha_{1}+\alpha_{2}+1\right)} .
\end{aligned}
$$

The proof of Theorem 2.6 is complete.

MISSOURI J. OF MATH. SCI., FALL 2015 


\section{H.-P. YIN AND F. QI}

Corollary 2.7. Under conditions of Theorem 2.6, if $p=\ell \leq \min \left\{q, \frac{q}{q-1}\right\}$, then we have

$$
\begin{aligned}
& \frac{1}{b-a} \int_{a}^{b} f(x) g(x) d x \\
& \leq \frac{1}{\left(\alpha_{1}+1\right)\left(\alpha_{2}+1\right)\left(\alpha_{1}+\alpha_{2}+1\right)} \\
& \times\left[\min \left\{M\left(a, b ; f^{p}, g^{q-p(q-1)}\right), M\left(b, a ; f^{p}, g^{q-p(q-1)}\right)\right\}\right]^{1 / q} \\
& \times\left[\min \left\{M\left(a, b ; f^{(q-p) /(q-1)}, g^{p}\right), M\left(b, a ; f^{(q-p) /(q-1)}, g^{p}\right)\right\}\right]^{1-1 / q} .
\end{aligned}
$$

Corollary 2.8. Under conditions of Theorem 2.6, when $\alpha_{1}=\alpha_{2}=m_{1}=$ $m_{2}=1$, we have

$$
\begin{gathered}
\frac{1}{b-a} \int_{a}^{b} f(x) g(x) d x \leq \frac{1}{6}\left[2 f^{p}(a) g^{q-\ell(q-1)}(a)+f^{p}(a) g^{q-\ell(q-1)}(b)\right. \\
\left.+f^{p}(b) g^{q-\ell(q-1)}(a)+2 f^{p}(b) g^{q-\ell(q-1)}(b)\right]^{1 / q}\left[2 f^{(q-p) /(q-1)}(a) g^{\ell}(a)\right. \\
\left.+f^{(q-p) /(q-1)}(a) g^{\ell}(b)+f^{(q-p) /(q-1)}(b) g^{\ell}(a)+2 f^{(q-p) /(q-1)}(b) g^{\ell}(b)\right]^{1-1 / q} .
\end{gathered}
$$

\section{ACKNOWLEDGEMENTS}

The authors would like to thank the anonymous referee for his/her kind and valuable comments on the original version of this manuscript.

This work was partially supported by the NNNSF of China under Grant No. 11361038 and by the Foundation of the Research Program of Science and Technology at Universities of Inner Mongolia Autonomous Region under Grant No. NJZY13159, China.

\section{REFERENCES}

[1] R.-F. Bai, F. Qi, and B.-Y. Xi, Hermite-Hadamard type inequalities for the $m$ - and $(\alpha, m)$-logarithmically convex functions, Filomat, 27.1 (2013), 1-7; http://dx.doi.org/10.2298/FIL1301001B.

[2] M. K. Bakula, M. E. Özdemir, and J. Pečarić, Hadamard type inequalities for $m$-convex and $(\alpha, m)$-convex functions, J. Inequal. Pure Appl. Math. 9.4 (2008), Article 96, 12 pages; http://www.emis.de/journals/JIPAM/article1032.html.

[3] S. S. Dragomir and G. Toader, Some inequalities for $m$-convex functions, Studia Univ. Babeş-Bolyai Math., 38.1 (1993), 21-28.

[4] V. G. Miheşan, A generalization of the convexity, Seminar on Functional Equations, Approx. Convex, Cluj-Napoca, 1993. (Romania)

[5] B. G. Pachpatte, On some inequalities for convex functions, RGMIA Res. Rep. Coll. 6 (2003), Article 1; http://rgmia.org/v6(E).php.

[6] Y. Shuang, H.-P. Yin, and F. Qi, Hermite-Hadamard type integral inequalities for geometric-arithmetically s-convex functions, Analysis (Munich) 33.2 (2013), 197208; http://dx.doi.org/10.1524/anly.2013.1192. 


\section{HERMITE-HADAMARD INEQUALITIES FOR CONVEX FUNCTIONS}

[7] G. Toader, Some generalizations of the convexity, Proceedings of the Colloquium on Approximation and Optimization, Univ. Cluj-Napoca, Cluj, 1985, 329-338.

[8] B.-Y. Xi and F. Qi, Hermite-Hadamard type inequalities for functions whose derivatives are of convexities, Nonlinear Funct. Anal. Appl., 18.2 (2013), 163-176.

[9] B.-Y. Xi and F. Qi, Some Hermite-Hadamard type inequalities for differentiable convex functions and applications, Hacet. J. Math. Stat., 42.3 (2013), 243-257.

[10] B.-Y. Xi and F. Qi, Some inequalities of Hermite-Hadamard type for h-convex functions, Adv. Inequal. Appl., 2.1 (2013), 1-15.

[11] B.-Y. Xi, Y. Wang, and F. Qi, Some integral inequalities of Hermite-Hadamard type for extended $(s, m)$-convex functions, Transylv. J. Math. Mechanics, 5.1 (2013), 6984.

[12] T.-Y. Zhang, A.-P. Ji, and F. Qi, Some inequalities of Hermite-Hadamard type for GA-convex functions with applications to means, Matematiche (Catania), 68.1 (2013), 229-239; http://dx.doi.org/10.4418/2013.68.1.17.

MSC2010: 26D15, 41A55

Key words and phrases: Hermite-Hadamard type inequality; $(\alpha, m)$-convex function; product; Hölder's integral inequality

College of Mathematics, Inner Mongolia University for Nationalities, Tongliao

City, Inner Mongolia Autonomous Region, 028043 China

E-mail address: hongpingyin@qq.com>

Department of Mathematics, College of Science, Tianjin Polytechnic University, Tianjin City, 300160 China

E-mail address: qifeng618@gmail.com

MISSOURI J. OF MATH. SCI., FALL 2015 